Article

\title{
Effects of Propagation of Narrow Bipolar Pulses, Generated by Compact Cloud Discharges, over Finitely Conducting Ground ${ }^{\dagger}$
}

\author{
Vernon Cooray ${ }^{1, *}$, M. Fernando ${ }^{2}$, L. Gunasekara ${ }^{2}$ and S. Nanayakkara ${ }^{2}$ \\ 1 Department of Engineering Sciences, Uppsala University, 75237 Uppsala, Sweden \\ 2 Department of Physics, University of Colombo, Colombo 03, Sri Lanka; \\ mahendra.fernando@gmail.com (M.F.); lasitha.niwanka@gmail.com (L.G.); \\ sankha.nanayakkara@sci.cmb.ac.lk (S.N.) \\ * Correspondence: vernon.cooray@angstrom.uu.se \\ $+\quad$ This paper is an extended version of a paper presented at the International Conference on Lightning \\ Protection, Shanghai, China, 11-18 October 2014.
}

Received: 19 April 2018; Accepted: 14 May 2018; Published: 17 May 2018

\begin{abstract}
Propagation effects on the narrow bipolar pulses (NBPs) or the radiation fields generated by compact cloud discharges as they propagate over finitely conducting ground are presented. The results were obtained using a sample of NBPs recorded with high time resolution from close thunderstorms in Sri Lanka. The results show that the peak amplitude and the temporal features such as the full width at half maximum (FWHM), zero-crossing time, and the time derivative of NBPs can be significantly distorted by propagation effects. For this reason, the study of peak amplitudes and temporal features of NBPs and the remote sensing of current parameters of compact cloud discharges should be conducted using NBPs recorded under conditions where the propagation effects are minimal.
\end{abstract}

Keywords: compact cloud discharges; narrow bipolar pulses; propagation effects; finitely conducting ground

\section{Introduction}

The knowledge concerning the characteristics of electromagnetic fields generated by lightning flashes is of importance in evaluating the interaction of these electromagnetic fields with electrical networks and in the remote sensing of lightning current parameters from the measured fields [1-4]. However, electromagnetic fields generated by lightning flashes change their character as they propagate over the ground surface due to selective attenuation of the high frequency signals by finitely conducting ground (i.e., propagation effects). Thus, depending on the distance of propagation and the conductivity of ground, the peak, the rise time, full width at half maximum (FWHM), zero-crossing time of the lightning generated electromagnetic fields and their time derivatives measured at a given distance from the lightning channel may deviate more or less depending on the conductivity from the values that would be present over perfectly conducting ground.

Most of the studies conducted so far on the propagation effects have concentrated on the radiation fields generated by return strokes [5]. Propagation effects on pulses generated by cloud flashes were investigated by Cooray [6]. Narrow bipolar pulses (NBPs) are radiation fields generated by short duration cloud discharges known as compact cloud discharges [7-15]. To the best of our knowledge, studies pertinent to the propagation effects on the NBPs are not available in the literature. In this paper, we will study the propagation effects caused by finitely conducting ground on the NBPs. 
Propagation effects are evaluated for both negative NBPs, positive NBPs, and derivatives of NBPs. The results are based on 100 negative NBPs, 29 positive NBPs, and 9 waveforms of measured derivatives of NBPs. Since NBPs are produced by electrical discharges taking place in the cloud the theory developed by Cooray [6] to calculate the propagation effects on pulses generated by cloud flashes will be utilized. The atmospheric sign convention is used in defining the polarity of the pulses.

Preliminary results of this study, based only on 27 negative NBPs, were reported previously by the same authors at the International Conference on Lightning Protection held in China, 2014.

\section{Theory and Methods}

The geometry of the situation under consideration is shown in Figure 1.

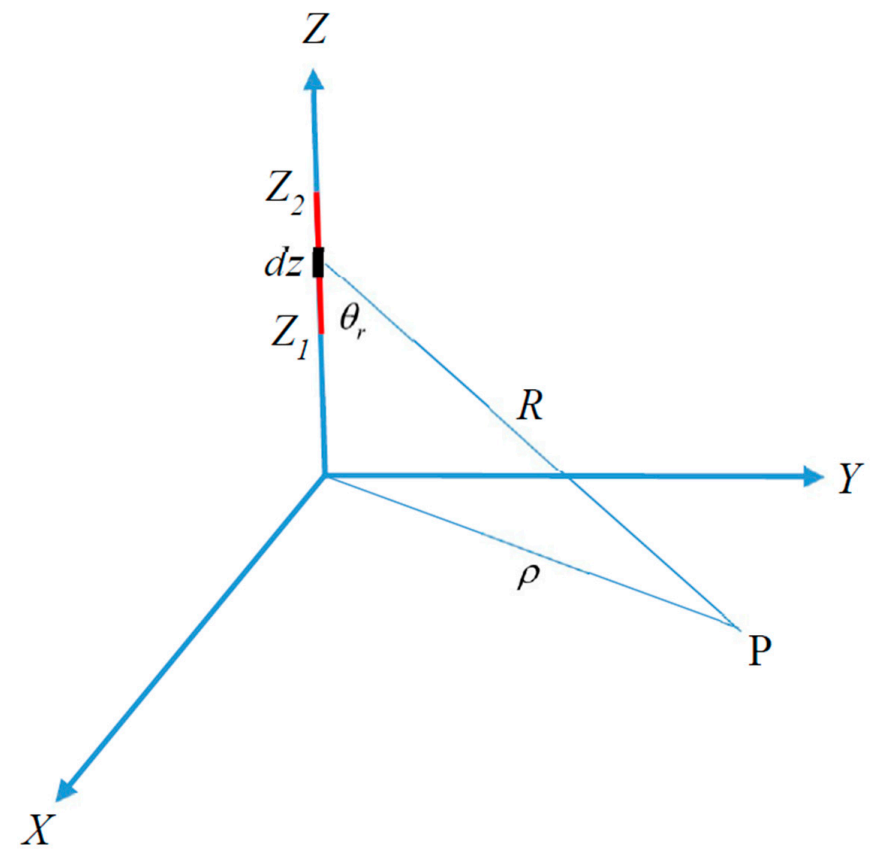

Figure 1. Geometry relevant to the calculation of propagation effects on electromagnetic fields generated by cloud flashes. The $X-Y$ plane represents the finitely conducting ground plane and the point of observation is located at ground level.

Let us assume that the channel of the compact cloud discharge is vertical. Let $Z_{1}$ be the height of origin of the discharge and $Z_{2}$ be the height where it was terminated. The vertical electric field at a point of observation located at ground level, caused by the current flowing along the discharge channel is given by

$$
e_{z}(j \omega, \rho)=\int_{Z_{1}}^{Z_{2}} \frac{I(z, j \omega)}{2 \pi \varepsilon_{0}}\left(\frac{3 \sin ^{2} \theta_{r}-2}{j \omega R^{3}}+\frac{3 \sin ^{2} \theta_{r}-2}{c R^{2}}+j \omega \frac{\sin ^{2} \theta_{r}}{c^{2} R} a(z, j \omega, \rho)\right) e^{-j \omega R / c} d z
$$

In the above equation, $j^{2}=-1, c$ is the speed of light in free space, $I(z, j \omega)$ is the current in the channel element $d z$ and $a(z, j \omega, \rho)$ is the attenuation function. The attenuation function is given by [6]

$$
a(z, j \omega, \rho)=\frac{1}{2}\left[\left(1+R_{v}\right)+\left(1-R_{v}\right) F(w, z)\right]
$$

In the above expression

$$
R_{v}=\frac{\cos \theta_{r}-\Delta_{0}}{\cos \theta_{r}+\Delta_{0}}
$$




$$
\begin{gathered}
\Delta_{0}=\frac{\gamma_{0}}{\gamma}\left[1-\frac{\gamma_{0}^{2}}{\gamma^{2}} \sin ^{2} \theta_{r}\right]^{1 / 2} \\
\gamma_{0}=\omega / c ; \quad \gamma=\gamma_{0} \sqrt{\varepsilon-j \sigma / \omega \varepsilon_{0}} \\
w=\frac{-j \gamma_{0} R}{2 \sin ^{2} \theta_{r}}\left[\cos \theta_{r}+\Delta_{0}\right]^{2} \\
F(w)=1-j(\pi w)^{1 / 2} e^{-w} \operatorname{erf} c\left(j w^{1 / 2}\right)
\end{gathered}
$$

In these equation, $\varepsilon$ is the relative dielectric constant, $\sigma$ is the conductivity of soil, and $\operatorname{erf} c$ is the complementary error function. The angle $\theta_{r}$ is defined in Figure 1.

Observe that the electric field given by Equation (1) is directed into the ground and, since we use atmospheric sign convention, it is assumed to be of positive polarity. Since the length of the channel of compact cloud discharges is no more than a few hundred meters (as inferred from the pulse durations) the attenuation function can be replaced by the function corresponding to height $Z_{1}$ i.e., $a\left(Z_{1}, j \omega, \rho\right)$. With this approximation, Equation (1) can be inverse Fourier transformed into time domain as

$$
\begin{aligned}
E_{z, c l}(t, \rho)=E_{z, s, c l}(t, \rho) & +E_{z, i, c l}(t, \rho) \\
& +\int_{0}^{t} E_{z, r, c l}(t-\tau, \rho) W\left(Z_{1}, t, \rho\right) d \tau
\end{aligned}
$$

In the above equation, $W\left(Z_{1}, t, \rho\right)$ is the inverse Fourier transformation of $a\left(Z_{1}, j \omega, \rho\right)$ and $E_{z, s, c l}(t, \rho), E_{z, i, c l}(t, \rho)$, and $E_{z, r, c l}(t, \rho)$ are the static, induction, and radiation field components, respectively, of the electric fields generated by the compact cloud discharge over perfectly conducting ground. These field components are given by

$$
\begin{gathered}
E_{z, s, c l}(t, \rho)=\int_{Z_{1}}^{Z_{2}} \frac{d z}{2 \pi \varepsilon_{0}}\left\{\frac{3 \sin ^{2} \theta_{r}-2}{R^{3}} \int_{0}^{t} i(z, \tau-R / c) d \tau\right\} \\
E_{z, i, c l}(t, \rho)=\int_{Z_{1}}^{Z_{2}} \frac{d z}{2 \pi \varepsilon_{o}} \frac{3 \sin ^{2} \theta_{r}-2}{c R^{2}} i(z, t-R / c) \\
E_{z, r, c l}(t, \rho)=\int_{Z_{1}}^{Z_{2}} \frac{d z}{2 \pi \varepsilon_{o}} \frac{\sin ^{2} \theta_{r}}{c^{2} R} \frac{\partial i(z, t-R / c)}{\partial t}
\end{gathered}
$$

In the case of pure radiation fields, Equation (8) reduces to

$$
E_{z, c l}(t, \rho)=\int_{0}^{t} E_{z, r, c l}(t-\tau, \rho) W\left(Z_{1}, t, \rho\right) d \tau
$$

Using the above technique, if the undistorted radiation fields generated by compact cloud discharges (i.e., NBPs) are available, they can be used in the above equation to evaluate the propagation effects.

\section{Results and Discussion}

In this study, the narrow bipolar pulses recorded from nearby thunderstorms in Sri Lanka, in May 2013, are used as the undistorted waveforms. In other words, we assume that the recorded waveforms are not distorted by propagation effects. The reason for this assumption is the following. The measuring station is located about $50 \mathrm{~m}$ from the Indian Ocean and the thunderstorms were located within about $20-80 \mathrm{~km}$ over the ocean (based on satellite images). Since the path of propagation of the waveforms is over salt water, the propagation effects on the sample of data selected in the analysis is 
minimal. The presence of $30 \mathrm{MHz}$ radiation in the recorded waveforms also confirms this assertion. The experimental set up used to record the data and the general features of the recorded NBPs were presented previously in Gunasekara et al. [15].

Since the recorded NBPs are pure and undistorted radiation fields, Equation (12) can be used to calculate the propagation effects. In order to calculate the propagation effects, the only other parameter that is necessary is the height of origin of the bipolar pulses. Unfortunately, the heights of origin of the narrow bipolar pulses are not known but they may occur at heights from about $5 \mathrm{~km}$ to $13 \mathrm{~km}$; as such, calculations are done for several heights of origin. The main features of propagation effects do not change significantly as this height is changed and results pertinent to $5 \mathrm{~km}$ height are presented in this publication.

As previously noted by Cooray [6], propagation effects are different for a source located at ground and for a source located at a certain height from ground level. In the case of a source located at ground level, the distance radiation field consists only of a ground wave. In the case of a source located above the ground level, the distance radiation field consists of a ground wave and a sky wave. It is the ground wave that is being attenuated by the finitely conducting ground. For this reason, in the case of a source located at a certain height from ground level, this is the only part of the distant radiation field which is affected by the finitely conducting ground. In order to illustrate this, assume that the source is excited by a current moment whose temporal variation can be represented by a ramp function. In this case, the radiation field over perfectly conducting ground will have the temporal shape of a step function. However, when the ground is finitely conducting this shape will be modified due to attenuation of high frequencies. The resulting radiation field signature at two distances over finitely conducting ground of $0.001 \mathrm{~S} / \mathrm{m}$ is shown in Figure 2. In the calculation, the height of the source from ground level was fixed at $5 \mathrm{~km}$. Observe that part of the radiation field still behaves as a step and this is the part associated with the sky wave. Note how the amplitude of the sky wave decreases with distance. Let us now consider the propagation effects on NBPs.

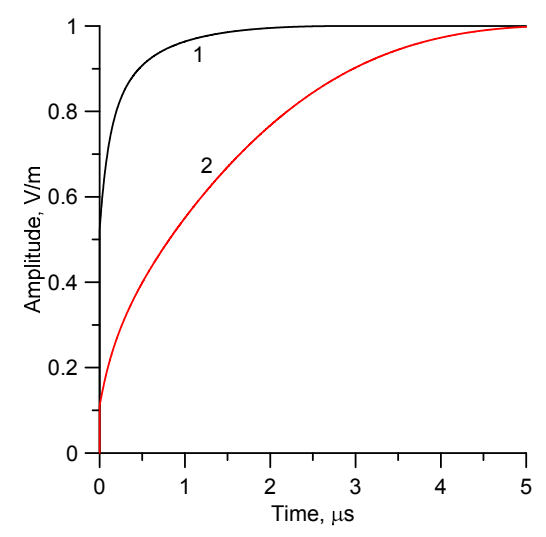

Figure 2. The variation of $E_{z, c l}(t, \rho)$ in Equation (12) when $E_{z, r, c l}(t, \rho)$ is a step function. In the calculation, the height of the source is at $5 \mathrm{~km}$ and $\rho=10 \mathrm{~km}$ for curve 1 and $\rho=100 \mathrm{~km}$ for curve 2. The conductivity of the ground was $0.001 \mathrm{~S} / \mathrm{m}$. Note the step at $t=0$ which is produced by the sky wave.

An example of a narrow bipolar pulse propagating $10 \mathrm{~km}, 50 \mathrm{~km}, 100 \mathrm{~km}$, and $200 \mathrm{~km}$ over finitely conducting ground of ground conductivity $0.001 \mathrm{~S} / \mathrm{m}$ is shown in Figure 3 . In this figure, the amplitude of the radiation field over perfectly conducting ground is normalized to unity. Moreover, in order to illustrate the propagation effects, the decrease of the radiation field with distance over perfectly conducting ground is removed from the data. That is, if there were no propagation effects all the waveforms would have an amplitude equal to unity. In the calculation, the height of origin of the pulse is assumed to be $5 \mathrm{~km}$. Observe that the propagation effects significantly distort the waveform. 
They reduce the peak amplitude of the pulse significantly. At the same time, the propagation effects lead to the increase in the zero-crossing time of the pulse.

The propagation effects on the derivative of a NBP is shown in Figure 4. Observe how the amplitude of the pulses associated with the derivative of the NBP decreases very rapidly with distance.

In order to study the attenuation of the peak amplitude of the NBPs due to propagation, let us define the attenuation coefficient $\mathrm{A}$ at any given distance as the ratio between the peak amplitude of the NBP over finitely conducting ground at that distance and the corresponding peak amplitude that would be present at the same distance over perfectly conducting ground. In other words, if the ground is perfectly conducting then $\mathrm{A}=1$ at any distance and it decreases with decreasing conductivity. In Figure 5, the variation of A for negative NBPs, positive NBPs, and for the derivatives of NBPs are depicted as a function of distance for $0.001 \mathrm{~S} / \mathrm{m}$ conductivity and for a source height of $5 \mathrm{~km}$. In the case of the derivatives of the NBPs, the value of A is estimated using the largest amplitude of the derivatives at the given distance. First, observe that the propagation effects can significantly decrease the peak amplitude of the NBPs and their derivatives are severely attenuated by the finitely conducting ground. The attenuation of the initial peak is larger the longer the distance of propagation and the smaller the conductivity of ground. For this reason, only the NBPs measured from nearby thunderstorms over good conducting ground should be used in gathering statistics on the peak amplitude of NBPs. As one can see from Figure 3, the same is true for the zero-crossing time of the NBPs. Note in Figure 3 how the zero-crossing time is increased by a factor of about 2 in the waveform that has propagated $200 \mathrm{~km}$ over finitely conducting ground.

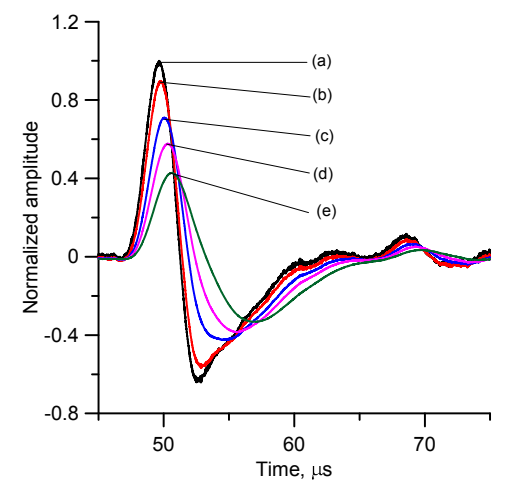

Figure 3. The effect of propagation on a narrow bipolar pulse (NBP). (a) The radiation field that would be present over perfectly conducting ground. The curves corresponding to the propagation over finitely conducting ground over distances of (b) $10 \mathrm{~km},(\mathbf{c}) 50 \mathrm{~km}$, (d) $100 \mathrm{~km}$, and (e) $200 \mathrm{~km}$. The conductivity of the ground is $0.001 \mathrm{~S} / \mathrm{m}$ and the height of the source of the NBP is $5 \mathrm{~km}$.

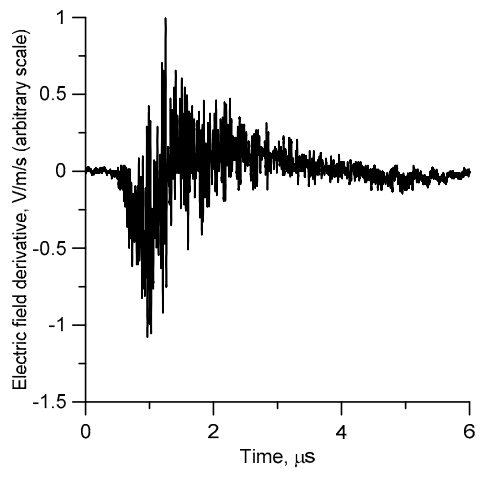

(a)

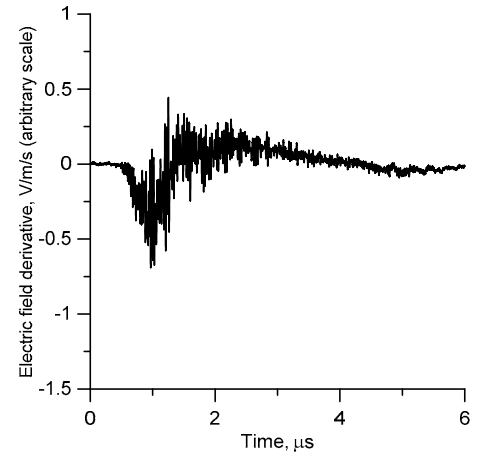

(b)

Figure 4. Cont. 


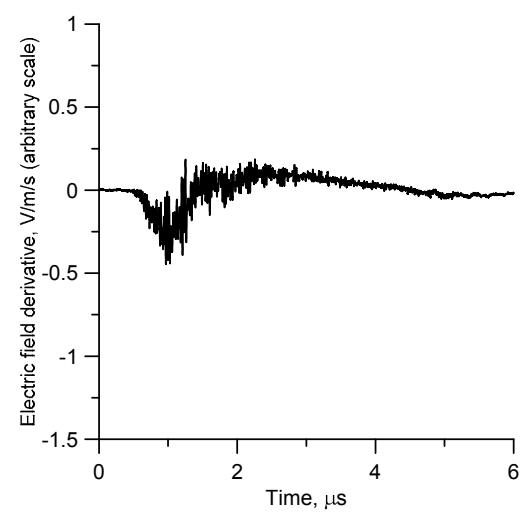

(c)

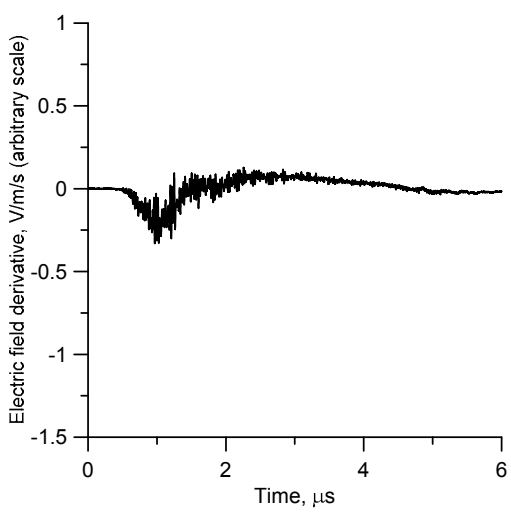

(d)

Figure 4. The propagation effects on the derivative of a typical NBP. (a) Undistorted derivative. (b) Derivative at $10 \mathrm{~km}$. (c) Derivative at $30 \mathrm{~km}$. (d) Derivative at $50 \mathrm{~km}$. The conductivity of the ground was $0.001 \mathrm{~S} / \mathrm{m}$. Note that the vertical scale is arbitrary, but it is proportional to the units $\mathrm{V} / \mathrm{m} / \mathrm{s}$.

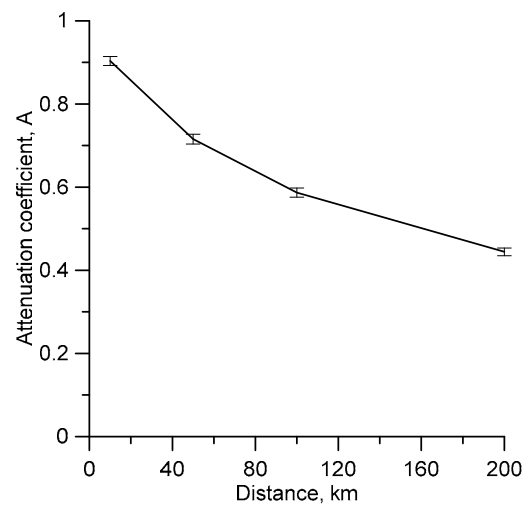

(a)

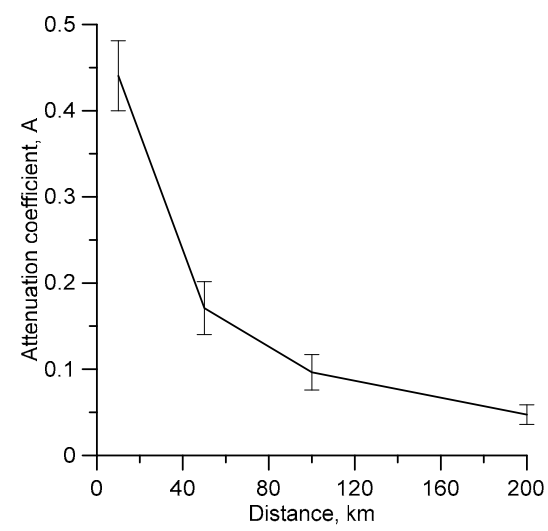

(c)

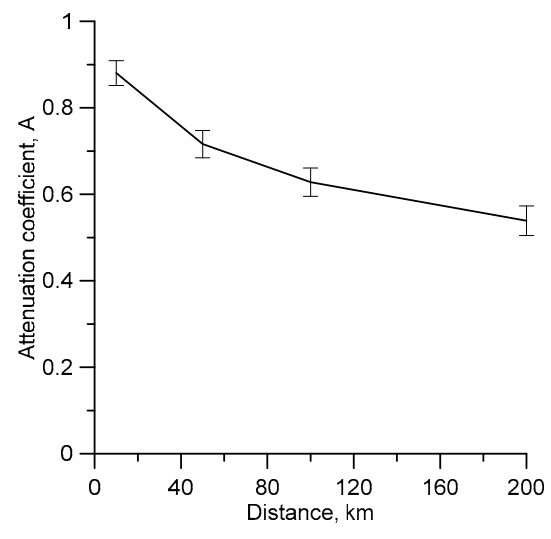

(b)

Figure 5. The variation of attenuation coefficient, A, of NBPs as a function of distance over finitely conducting ground. (a) Negative NBP. (b) Positive NBP. (c) Derivative of NBP. The error bars indicate the standard deviation. The conductivity of the ground is $0.001 \mathrm{~S} / \mathrm{m}$ and the height of the source is $5 \mathrm{~km}$. 
It is interesting to note that NBPs were first detected by LeVine [7] as the events that produced the highest amount of high frequency (HF) radiation among various radiation field pulses produced by lightning flashes. Now, a feature of the NBP that distinguishes it from other lightning events is the signature of its derivative. Its derivative contains a large amount of high frequency oscillations (see Figure 4) and it is possible that the HF radiation detected by LeVine [7] was generated by the same process that gives rise to these rapid oscillations in the derivatives of the NBPs. However, these high frequency oscillations in the derivative will rapidly decrease as the NBP propagates over finitely conducting ground and as a result one may not be able to distinguish these pulses from other radiation field pulses generated by cloud flashes when they have propagated significant distances over finitely conducting ground. Thus, in order to separate NBPs from other cloud events they have to be measured in such a way so that the propagation effects are minimal and the main features that distinguish them from other lightning radiation pulses are retained.

The propagation effects not only reduce the amplitudes but also modify the shape of the waveforms. As one can see from the results presented in Figure 3, the zero-crossing time, the full width at half maximum (FWHM), and the ratio of the initial peak to the opposite overshoot also change as the waveform propagates along finitely conducting ground. Figure 6 shows how the average zero-crossing time of the negative narrow bipolar pulses (based on 100 waveforms) varies as the pulses propagate over finitely conducting ground. Figure 7 depicts how the FWHM is increased due to propagation effects. Results are shown in Figures 6 and 7 for two conductivities: $0.001 \mathrm{~S} / \mathrm{m}$ and $0.0005 \mathrm{~S} / \mathrm{m}$. The results show that the zero-crossing time of the undistorted narrow bipolar pulses is about $4 \mu \mathrm{s}$, but it could increase to about 10-15 $\mu$ s after about 100 to $200 \mathrm{~km}$ propagation over finitely conducting ground. The average FWHM of the undistorted NBPs is about $2.1 \mu \mathrm{s}$ and it can increase to about $3 \mu$ s due to propagation effects. The average values of the undistorted zero-crossing time and the FWHM are in agreement with the results presented by Gunasekara et al. [15]. These features of the propagation effects have to be considered when remote sensing is used to measure the currents in compact cloud flashes using the features of narrow bipolar pulses.

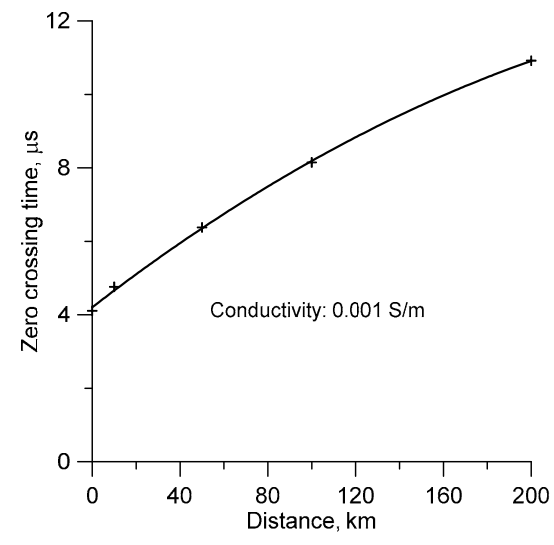

(a)

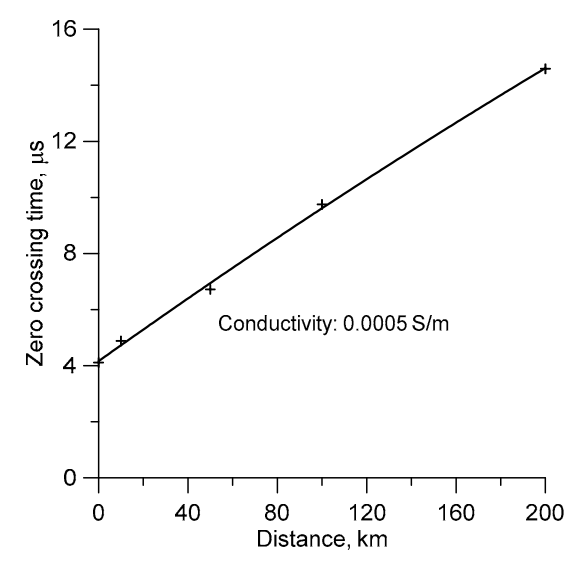

(b)

Figure 6. Variation of the zero-crossing time as the negative NBPs propagate over finitely conducting ground of two different conductivities: (a) $0.001 \mathrm{~S} / \mathrm{m}$, (b) $0.0005 \mathrm{~S} / \mathrm{m}$. The value at distance zero corresponds to the parameter pertinent to undistorted waveforms. In the calculation, the source height was fixed at $5 \mathrm{~km}$. 


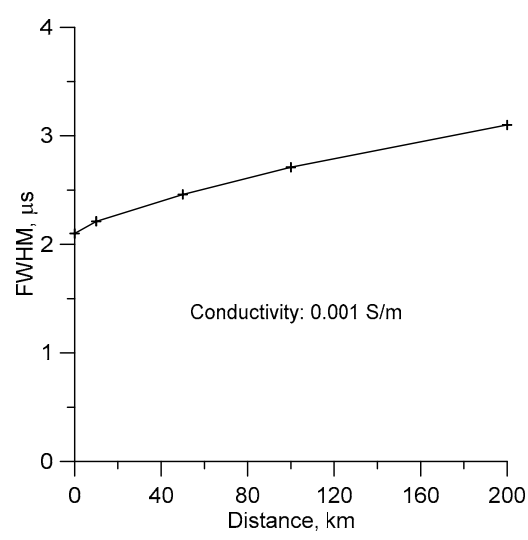

(a)

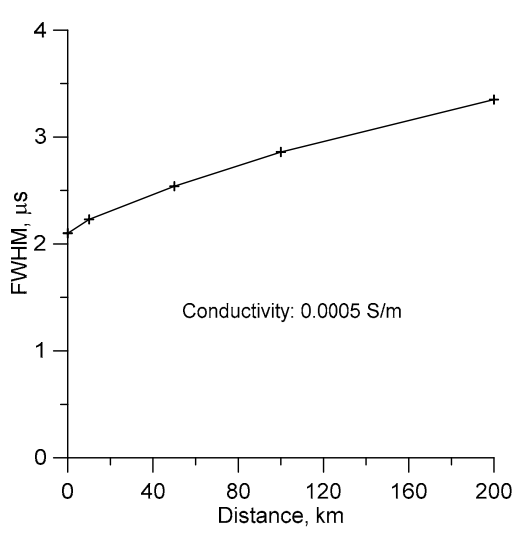

(b)

Figure 7. Variation of the full width at half maximum (FWHM) as the negative NBPs propagate over finitely conducting ground of two different conductivities: (a) $0.001 \mathrm{~S} / \mathrm{m},(\mathbf{b}) 0.0005 \mathrm{~S} / \mathrm{m}$. The value at distance zero corresponds to the parameter pertinent to undistorted waveforms. In the calculation, the source height was fixed at $5 \mathrm{~km}$.

Another waveform parameter that is affected by propagation effects is the ratio of the initial peak to the peak of the opposite overshoot. Figure 8 depicts how this parameter varies as the negative NBPs propagate over finitely conducting ground. Note that the way in which this parameter varies with distance is rather complex. This ratio depends on how the two peaks (initial peak and the opposite overshoot) are attenuated with distance. This in turn depends on the frequency content or the time width associated with the peaks. According to the results given in Figure 8, initially the opposite overshoot is attenuated more than the initial peak, but once the propagation distance becomes $50 \mathrm{~km}$ or so the attenuation of the initial peak becomes larger than the opposite overshoot. For this reason, for distances larger than about $50 \mathrm{~km}$ the ratio starts to decrease with distance.

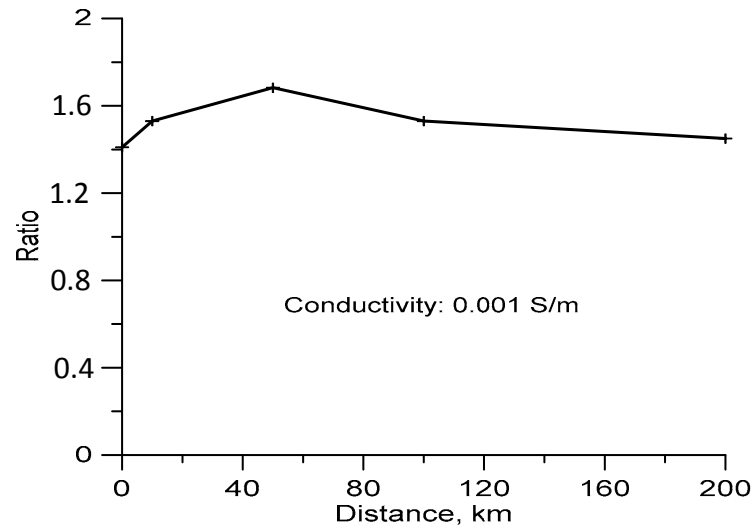

(a)

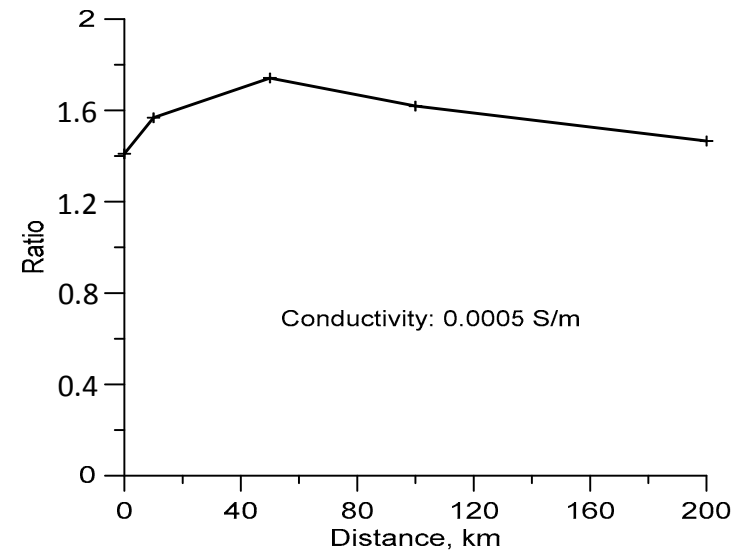

(b)

Figure 8. Variation of the ratio of the initial peak to the opposite overshoot of the negative NBPs as they propagate over finitely conducting ground of two different conductivities: (a) $0.001 \mathrm{~S} / \mathrm{m}$; (b) $0.0005 \mathrm{~S} / \mathrm{m}$. The value at distance zero corresponds to the parameter pertinent to undistorted waveforms. In the calculation, the source height was fixed at $5 \mathrm{~km}$. 


\section{Conclusions}

The results presented in this paper show that, due to their narrow width, the peak amplitude, the zero-crossing time, the FWHM, and the time derivatives of NBPs or the radiation fields from compact cloud discharges are significantly distorted by propagation effects. Any study designed to collect the statistical data on either the peak amplitude, the zero-crossing time, FWHM, or the time derivatives of these pulses should be conducted over ground planes of high conductivity utilizing nearby thunderstorms. Moreover, in any study pertinent to the remote sensing of currents of compact cloud discharges using NBPs, the data sample has to be recorded in such a way to minimize the propagation effects.

Author Contributions: The study was completed with cooperation between all authors. The lightning research group in Sri Lanka (M.F., S.N., L.G.) recorded the data and V.C. conducted the analysis of propagation effects and wrote the paper.

Funding: This work was funded by the University of Colombo research grant AP/3/2012/CG/24. Vernon Cooray's participation in the project is funded by Grants 621-2003-3465 and 621-2006-4299 from the Swedish National Research Council (VR).

Conflicts of Interest: The authors declare no conflict of interest.

\section{References}

1. Cooray, V.; de La Rosa, F. Shapes and amplitudes of the initial peaks of lightning-induced voltage in power lines over finitely conducting earth: Theory and comparison with experiment. IEEE Trans. Antennas Propag. 1986, 34, 88-92. [CrossRef]

2. Rachidi, F.; Nucci, C.A.; Ianoz, M. Transient analysis of multiconductor lines above a lossy ground. IEEE Trans. Power Deliv. 1999, 14, 294-302. [CrossRef]

3. Nucci, C.A.; Rachidi, F.; Ianoz, M.V.; Mazzetti, C. Influence of a lossy ground on lightning induced voltages on overhead lines. IEEE Trans. Electromagn. Compat. 1993, 35, 75-86. [CrossRef]

4. Cooray, V:; Orville, R. The effects of variation of current amplitude, current risetime and return stroke velocity along the return stroke channel on the electromagnetic fields generated by return strokes. J. Geophys. Res. 1990, 95, 18617-18630. [CrossRef]

5. Cooray, V. On the various approximations to calculate lightning return stroke generated electric and magnetic fields over finitely conducting ground. In Lightning Electromagnetics; Cooray, V., Ed.; IET: Stevenage, UK, 2012.

6. Cooray, V. Propagation effects on radiation field pulses generated by cloud flashes. J. Atmos. Sol. Terr. Phys. 2007, 69, 1397-1406. [CrossRef]

7. LeVine, D.M. Sources of strongest RF radiation from lightning. J. Geophys. Res. 1980, 85, 4091-4095. [CrossRef]

8. Willett, J.C.; Bailey, J.C.; Krider, E.P. A class of unusual lightning electric field waveforms with very strong high-frequency radiation. J. Geophys. Res. 1989, 94, 16255-16267. [CrossRef]

9. Medelius, P.J.; Thomson, E.M.; Pierce, J.S. E and DE/DT wave shapes for narrow bipolar pulses in intracloud lightning. In Proceedings of the International Aerospace and Ground Conference on Lightning and Static Electricity, Atlantic City, NY, USA, 6-8 October 1992; NASA Conference Publication: Washington, DC, USA, 1992.

10. Smith, D.A.; Shao, X.M.; Holden, D.N.; Rhodes, C.T.; Brook, M.; Krehbiel, P.R.; Stanley, M.; Rison, W.; Thomas, R.J. A distinct class of isolated intracloud lightning discharges and their associated radio emissions. J. Geophys. Res. 1999, 104, 4189-4212. [CrossRef]

11. Smith, D.A.; Heavner, M.J.; Jacobson, A.R.; Shao, X.M.; Massey, R.S.; Sheldon, R.J.; Weins, K.C. A method for determining intracloud lightning and ionospheric heights from VLF/LF electric field records. Radio Sci. 2004, 39. [CrossRef]

12. Jacobson, A.R.; Heavner, M.J. Comparison of narrow bipolar events with ordinary lightning as proxies for severe convection. Mon. Weather Rev. 2005, 133, 1144-1154. [CrossRef]

13. Nag, A.; Rakov, V.A.; Tsalikis, D.; Cramer, J.A. On phenomenology of compact intracloud lightning discharges. J. Geophys. Res. 2010, 115. [CrossRef] 
14. Ahmad, N.A.; Fernando, M.; Bahaudin, Z.A.; Cooray, V.; Ahmad, H.; Malek, Z.A. Characteristics of narrow bipolar pulses observed in Malaysia. J. Atmos. Sol. Terr. Phys. 2010, 72, 534-540. [CrossRef]

15. Gunasekara, T.A.L.N.; Fernando, M.; Sonnadara, U.; Cooray, V. Characteristics of Narrow Bipolar Pulses observed from lightning in Sri Lanka. J. Atmos. Sol. Terr. Phys. 2016, 138, 66-73. [CrossRef]

(c) 2018 by the authors. Licensee MDPI, Basel, Switzerland. This article is an open access article distributed under the terms and conditions of the Creative Commons Attribution (CC BY) license (http://creativecommons.org/licenses/by/4.0/). 